\title{
Quantum Heisenberg Antiferromagnet on Frustrated Bilayer Lattice in Strong Magnetic Fields
}

\author{
O. DerzhKo ${ }^{a}$, J. Richter ${ }^{b}$ And T. KROKhMAlskiI ${ }^{a}$ \\ ${ }^{a}$ Institute for Condensed Matter Physics, NASU \\ 1 Svientsitskii Street, L'viv-11, 79011, Ukraine \\ ${ }^{b}$ Institut für Theoretische Physik, Universität Magdeburg \\ P.O. Box 4120, 39016 Magdeburg, Germany
}

\begin{abstract}
In the present paper we consider the low-temperature thermodynamic properties of the quantum Heisenberg antiferromagnet on the frustrated bilayer lattice in strong magnetic fields.Under certain conditions the isolated localized magnon states (singlets on vertical bonds; simultaneous occupation of neighboring vertical bonds is forbidden) are the ground states which dominate thermodynamics and induce a behavior inherent in the well known model of hard squares on a square lattice. We focus on magnetic properties discussing the magnetization and the susceptibility of the spin model.
\end{abstract}

PACS numbers: 75.10.Jm, 75.45.+j, 75.50.Ee

\section{Introduction}

Antiferromagnetically interacting quantum Heisenberg spins on frustrated lattices (like pyrochlore, kagomé, checkerboard etc.) attract much attention nowadays,since they may provide realization of exotic states of matter. Application of an external magnetic field to a frustrated quantum Heisenberg antiferromagnet may lead to further intricate phenomena. Interestingly, some lattice geometries support localized magnon states which are located within a restricted area of the lattice rather than are extended all over the lattice [1] (for review see Ref. [2]). These states may dominate the low-temperature thermodynamics around the saturation field and may lead to a universal behavior which is amenable for rigorous analysis.

In this paper,we consider $N$ spins $s=1 / 2$ on a frustrated bilayer lattice (see Fig. 1) with the Heisenberg Hamiltonian

$$
H=\sum_{(n m)} J_{n m} \boldsymbol{s}_{n} \cdot \boldsymbol{s}_{m}-h S^{z} .
$$

Here the sum runs over the bonds connecting the neighboring sites, $J_{n m}>0$ may 

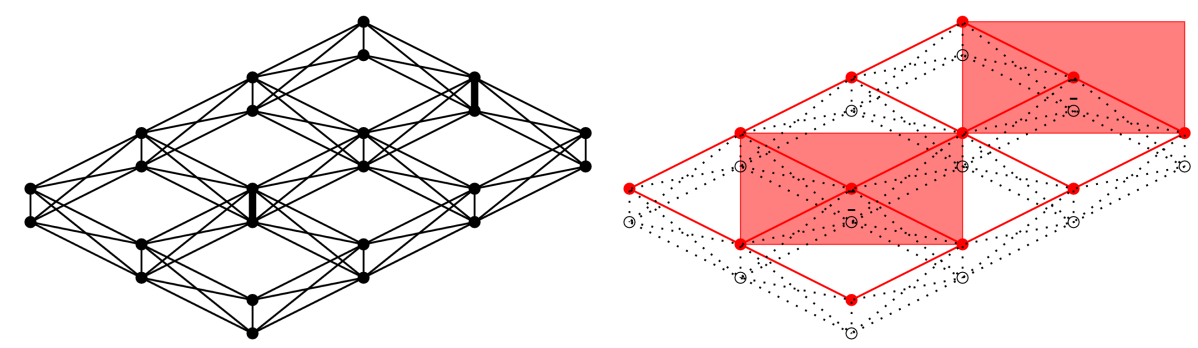

Fig. 1. Left: the frustrated bilayer lattice. The vertical bonds have the strength $J_{2}$ whereas all other bonds have the strength $J_{1}$. Right: The auxiliary model of hard squares on a square lattice, which emerges in a description of low-energy physics of the spin model in strong magnetic fields. The localized magnon located on vertical bonds are denoted by the bold lines in the left part; the hard squares which correspond to the occupied vertical bonds are shown in the right part.

acquire two values, $J_{2}$ for vertical bonds and $J_{1}$ for all other bonds, $S^{z}=\sum_{n} s_{n}^{z}$ is the $z$-component of the total spin. Since $S^{z}$ commutes with $H$ we may consider the eigenstates of $H$ in the subspaces with different $S^{z}=N / 2, N / 2-1, N / 2-2, \ldots$

\section{Theory and results}

Let us assume at first $h=0$. The fully polarized state $|\uparrow \ldots \uparrow\rangle$ with the energy $E_{\mathrm{FM}}$ is the eigenstate of $H$ in the subspace with $S^{z}=N / 2$. The singlet which is localized on a vertical bond in a ferromagnetic environment $|1 \mathrm{~lm}\rangle=$ $|\operatorname{lm}\rangle_{v}|\uparrow \ldots \uparrow\rangle_{e},|\operatorname{lm}\rangle_{v}=(1 / \sqrt{2})\left(\left|\uparrow_{u} \downarrow_{l}\right\rangle-\left|\downarrow_{u} \uparrow_{l}\right\rangle\right)_{v}$ (here $u$ and $l$ denote the sites of the upper and lower plane which are connected by the vertical bond enumerated by $v$ ) is the eigenstate of $H$ in the subspace with $S^{z}=N / 2-1$ with the energy $E_{\mathrm{FM}}-\epsilon_{1}, \epsilon_{1}=J_{2}+4 J_{1}$. It can be shown [3] that if $J_{2}>4 J_{1}$ this is the lowest-energy eigenstate separated by a gap from other states in the subspace with $S^{z}=N / 2-1$ with the degeneracy $g_{N}(1)=N / 2$ (the localized magnon can be placed on any of $N / 2$ vertical bonds). In the subspace with $S^{z}=N / 2-2$ two isolated localized magnons with the energy $E_{\mathrm{FM}}-2 \epsilon_{1}$ are the lowest-energy eigenstates of $H$ separated from other states by a gap [3]. Since a localized magnon state implies that the neighboring sites are fully polarized the localized magnons cannot be placed on neighboring vertical bonds and hence hard-square rule emerges (see Fig. 1). The degeneracy $g_{N}(2)$ equals to the canonical partition function of 2 hard squares on a square lattice of $\mathcal{N}=N / 2$ sites (see Fig. 1, right part). For the subspaces with $S^{z}=N / 2-3, \ldots, N / 2-N / 4$ we find the lowest-energy eigenstates with the energies $E_{\mathrm{FM}}-3 \epsilon_{1}, \ldots, E_{\mathrm{FM}}-(N / 4) \epsilon_{1}$ separated by a gap from other higher energy states and the degeneracies $g_{N}(3), \ldots, g_{N}(N / 4)$ equal to the canonical partition functions of $3, \ldots, N / 4$ hard squares on a square lattice of $\mathcal{N}$ sites. Numerics for finite systems up to $N=64$ confirm this spectroscopic analysis $[3,2]$. Moreover, the conclusions derived using the concept of localized magnons may be reproduced for the frustrated bilayer (but not, e.g., for the kagomé or 
checkerboard lattice) in a different way noticing that this model falls into a class of models with local conservation laws [4]. The localized magnons are linearly independent in the subspaces with $S^{z}=N / 2-1, \ldots, N / 4[5]$.

Let us consider now the case $h>0$. Obviously, for the saturation field $h_{1}=\epsilon_{1}$ the (ground-state) energies of the localized magnon states with different $S^{z}=N / 2, N / 2-1, \ldots, N / 4$ become the same and a huge degeneracy emerges. We wish to calculate the contribution of the localized magnons to thermodynamics at low temperatures (less than the energy gap between the localized magnon states and the higher energy states which depends on $J_{2} / J_{1}>4$ ) around $h_{1}$. Their contribution can be written in the form

$$
Z(T, h, N)=\exp \left(-\frac{E_{\mathrm{FM}}-h N / 2}{T}\right) \sum_{n=0}^{N / 4} g_{N}(n) \exp \left(\frac{\mu n}{T}\right) \propto \Xi(T, \mu, \mathcal{N}),
$$

where $\mu=h_{1}-h$ and $\Xi(T, \mu, \mathcal{N})$ is the grand-canonical partition function of the hard-square model. Eq. (2) implies a order-disorder phase transition inherent in the hard-square model at $z_{\mathrm{c}}=\exp \left(\mu_{\mathrm{c}} / T\right)=3.7962 \ldots[6,7]$ between the lowdensity phase $\left(z<z_{\mathrm{c}}\right)$, in which the $A$ - and $B$-sublattices of the underlying square lattice are equally occupied,and the high-density phase $\left(z>z_{\mathrm{c}}\right)$, in which one of the sublattices becomes more occupied than the other one. The phase transition belongs to the two-dimensional Ising universality class.

We focus on the magnetic properties of the spin model (1) at low temperatures around $h_{1}$ when a description based on (2) holds. For the underlying square lattice we introduce the sublattice magnetizations $\left\langle S_{A}^{z}\right\rangle=N / 4-\overline{n_{\mathrm{A}}}$ and $\left\langle S_{B}^{z}\right\rangle=$ $N / 4-\overline{n_{B}}(\langle(\ldots)\rangle$ denotes thermodynamic averaging for the spin model, $\overline{(\ldots)}$ denotes grand-canonical averaging for the hard-square model). We note that while the total magnetization $M=\left\langle S^{z}\right\rangle=\left\langle S_{\mathrm{A}}^{z}\right\rangle+\left\langle S_{\mathrm{B}}^{z}\right\rangle$ is related to the average number of hard squares $\bar{n}=\overline{n_{\mathrm{A}}}+\overline{n_{\mathrm{B}}}$, namely, $M=N / 2-\bar{n}, \bar{n}=T \partial \ln \Xi(T, \mu, \mathcal{N}) / \partial \mu$, the value of the staggered magnetization $\left|M_{\mathrm{s}}\right|=\left|\left\langle S_{\mathrm{A}}^{z}\right\rangle-\left\langle S_{\mathrm{B}}^{\mathrm{Z}}\right\rangle\right|$ is related to the order parameter for the hard-square model $\eta=\left|\overline{n_{\mathrm{A}}}-\overline{n_{\mathrm{B}}}\right| /(N / 4)$, namely, $\left|M_{\mathrm{s}}\right|=\eta N / 4$. The associated susceptibilities of the spin model $\chi^{\mathrm{sp}}=\partial M / \partial h$ and $\chi_{\mathrm{s}}^{\mathrm{sp}}=\partial M_{\mathrm{s}} / \partial h$ are related to the compressibility $\kappa$ and the staggered susceptibility $\chi$ of the hard-square model. Borrowing the broad knowledge from the theory of the hard-square model $[6,7]$ we may predict the following features of the frustrated bilayer at low temperatures around $h_{1}$. Let us consider the system at fixed $h$ slightly below $h_{1}$ (e.g., $h=0.98 h_{1}$ ). While temperature increasing crosses the value $T_{\mathrm{c}}(h) \approx\left(h_{1}-h\right) / 1.3340$ the system exhibits the two-dimensional Ising type phase transition: $M(T)$ has an inflection point, $\chi^{\mathrm{sp}}(T)$ exhibits a maximum, $\left|M_{\mathrm{s}}(T)\right|$ vanishes with exponent $\beta=1 / 8, \chi_{\mathrm{s}}^{\mathrm{sp}}(T)$ shows a divergency with the exponent $\gamma=7 / 4$. Moreover,the temperature dependence of the specific heat $C(T)$ exhibits a logarithmic singularity whereas the correlation length $\xi(T)$ diverges with exponent $\nu=1$. Let us note, this is not in contradiction with the Mermin-Wagner theorem, since only a discrete symmetry is broken spontaneously. 
With respect to experimental confirmations it is necessary to have a corresponding substance with an accessible value of the saturation field $h_{1}$. Moreover, since the elaborated arguments hold only for the adopted "ideal relations" between the exchange bonds it would be desirable to go beyond this assumption. From numerical studies for a similar one-dimensional frustrated quantum antiferromagnet [8] we may expect that the predicted effects of the localized magnon states should survive even for small deviations from the "ideal geometry" of the frustrated bilayer lattice.

\section{Acknowledgments}

O.D. is indebted to Magdeburg University for hospitality in the summer of 2007 .

\section{References}

[1] J. Schnack, H.-J. Schmidt, J. Richter, J. Schulenburg, Eur. Phys. J. B 24, 475 (2001); J. Schulenburg, A. Honecker, J. Schnack, J. Richter, H.-J. Schmidt, Phys. Rev. Lett. 88, 167207 (2002).

[2] J. Richter, J. Schulenburg, A. Honecker, in: Quantum Magnetism, Lecture Notes in Physics, Vol. 645, Eds. U. Schollwöck, J. Richter, D.J.J. Farnell, R.F. Bishop, Springer, Berlin 2004, p. 85; J. Richter, Fiz. Nizkikh Temp. (Kharkiv) 31, 918 (2005); M.E. Zhitomirsky, H. Tsunetsugu, Prog. Theor. Phys. Suppl. 160, 361 (2005); O. Derzhko, J. Richter, A. Honecker, H.-J. Schmidt, arXiv:condmat/0612281.

[3] J. Richter, O. Derzhko, T. Krokhmalskii, Phys. Rev. B 74, 144430 (2006).

[4] A. Honecker, F. Mila, M. Troyer, Eur. Phys. J. B 15, 227 (2000).

[5] H.-J. Schmidt, J. Richter, R. Moessner, J. Phys. A 39, 10673 (2006).

[6] R.J. Baxter, Exactly Solved Models in Statistical Mechanics, Academic Press, London 1982.

[7] H.C.M. Fernandes, J.J. Arenzon, Y. Levin, J. Chem. Phys. 126, 114508 (2007).

[8] O. Derzhko, J. Richter, Phys. Rev. B 70, 104415 (2004). 